\title{
The Performance of Emotion Classifiers for Children With Parent-Reported Autism: Quantitative Feasibility Study
}

Haik Kalantarian $^{1,2}$, BSc, MS, DPhil; Khaled Jedoui ${ }^{3}$, BSc; Kaitlyn Dunlap ${ }^{1,2}$, BA, MRes; Jessey Schwartz ${ }^{1,2}$, BA; Peter Washington $^{1,2}$, BA, MSc; Arman Husic ${ }^{1,2}$, BSc; Qandeel Tariq ${ }^{1,2}$, BSc, MSci; Michael Ning ${ }^{1,2}$, BSc; Aaron Kline $^{1,2}$, BSc; Dennis Paul Wall ${ }^{1,2,4}$, BSc, MSc, DPhil

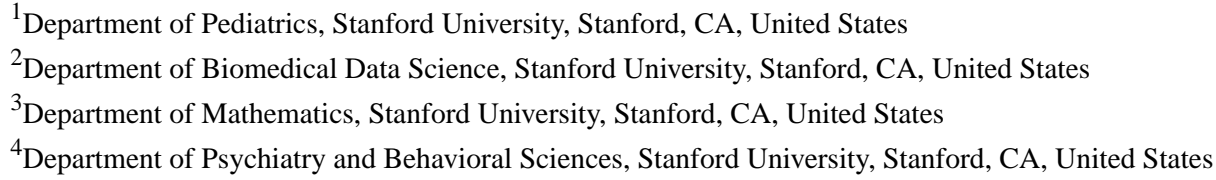

\section{Corresponding Author:}

Dennis Paul Wall, BSc, MSc, DPhil

Department of Pediatrics

Stanford University

450 Serra Mall

Stanford, CA, 94305

United States

Phone: 1 (650) 7232300

Email: dpwall@stanford.edu

\section{Abstract}

Background: Autism spectrum disorder (ASD) is a developmental disorder characterized by deficits in social communication and interaction, and restricted and repetitive behaviors and interests. The incidence of ASD has increased in recent years; it is now estimated that approximately 1 in 40 children in the United States are affected. Due in part to increasing prevalence, access to treatment has become constrained. Hope lies in mobile solutions that provide therapy through artificial intelligence (AI) approaches, including facial and emotion detection AI models developed by mainstream cloud providers, available directly to consumers. However, these solutions may not be sufficiently trained for use in pediatric populations.

Objective: Emotion classifiers available off-the-shelf to the general public through Microsoft, Amazon, Google, and Sighthound are well-suited to the pediatric population, and could be used for developing mobile therapies targeting aspects of social communication and interaction, perhaps accelerating innovation in this space. This study aimed to test these classifiers directly with image data from children with parent-reported ASD recruited through crowdsourcing.

Methods: We used a mobile game called Guess What? that challenges a child to act out a series of prompts displayed on the screen of the smartphone held on the forehead of his or her care provider. The game is intended to be a fun and engaging way for the child and parent to interact socially, for example, the parent attempting to guess what emotion the child is acting out (eg, surprised, scared, or disgusted). During a 90-second game session, as many as 50 prompts are shown while the child acts, and the video records the actions and expressions of the child. Due in part to the fun nature of the game, it is a viable way to remotely engage pediatric populations, including the autism population through crowdsourcing. We recruited 21 children with ASD to play the game and gathered 2602 emotive frames following their game sessions. These data were used to evaluate the accuracy and performance of four state-of-the-art facial emotion classifiers to develop an understanding of the feasibility of these platforms for pediatric research.

Results: All classifiers performed poorly for every evaluated emotion except happy. None of the classifiers correctly labeled over $60.18 \%$ (1566/2602) of the evaluated frames. Moreover, none of the classifiers correctly identified more than $11 \%(6 / 51)$ of the angry frames and 14\% (10/69) of the disgust frames.

Conclusions: The findings suggest that commercial emotion classifiers may be insufficiently trained for use in digital approaches to autism treatment and treatment tracking. Secure, privacy-preserving methods to increase labeled training data are needed to boost the models' performance before they can be used in AI-enabled approaches to social therapy of the kind that is common in autism treatments. 
(JMIR Ment Health 2020;7(4):e13174) doi: 10.2196/13174

\section{KEYWORDS}

mobile phone; emotion; autism; digital data; mobile app; mHealth; affect; machine learning; artificial intelligence; digital health

\section{Introduction}

\section{Background}

Autism spectrum disorder (ASD) is a neurodevelopmental disorder characterized by stereotyped and repetitive behaviors and interests as well as deficits in social interaction and communication [1]. In addition, autistic children struggle with facial affect and may express themselves in ways that do not closely resemble those of their peers [2-4]. The incidence of ASD has increased in recent years; it is now estimated that approximately 1 in 40 children in the United States is affected by this condition [5]. Although autism has no cure, there is strong evidence that suggests early intervention can improve speech and communication skills [6].

Common approaches to autism therapy include applied behavior analysis (ABA) and the early start Denver model (ESDM). In ABA therapy, the intervention is customized by a trained behavioral analyst to specifically suit the learner's skills and deficits [7]. The basis of this program is a series of structured activities that emphasize the development of transferable skills to the real world. Similarly, naturalistic developmental behavioral interventions such as ESDM support the development of core social skills through interactions with a licensed behavioral therapist while emphasizing joint activities and interpersonal exchange [8]. Both treatment types have been shown to be safe and effective, with their greatest impact potential occurring during early intervention at younger ages [9-11].

Despite significant progress in understanding this condition in recent years, imbalances in coverage and barriers to diagnosis and treatment remain. In developing countries, studies have noted a lack of trained health professionals, inconsistent treatments, and an unclear pathway from diagnosis to intervention [12-14]. Within the United States, research has shown that children in rural areas receive diagnoses approximately 5 months later than children living in cities [15]. Moreover, it has been observed that children from families near the poverty line receive diagnoses almost a full year later than those from higher-income families. Data-driven approaches have estimated that over $80 \%$ of US counties contain no diagnostic autism resources [16]. Even months of delayed access to therapy can limit the effectiveness of subsequent behavioral interventions [15]. Alternative solutions that can ameliorate some of these challenges could be derived from digital and mobile tools. For example, we developed a wearable system using Google Glass that leverages emotion classification algorithms to recognize the facial emotion of a child's conversation partner for real-time feedback and social support and showed treatment efficacy in a randomized clinical trial [17-25].

Various cloud-based emotion classifiers may help the value and reach of mobile tools and solutions. These include four commercially available systems: Microsoft Azure Emotion application programming interface (API) [26], Amazon Rekognition [27], Google Cloud Vision [28], and Sighthound [29]. Whereas most implementations of these emotion recognition APIs are proprietary, these algorithms are typically trained using large facial emotion datasets such as the Cohn-Kanade database [30] and Belfast-Induced Natural Emotion Database [31], which have few examples of children. Due to this bias in labeled examples, it is possible that these models do not generalize well to the pediatric population, including children with developmental delays such as autism, which is evaluated in this study. This study puts the disparity to test. To do so, we use our mobile game Guess What? [32-35]. This game (native to Android [36] and iOS [37] platforms) fosters engagement between the child and their social partner, such as a parent, through charades-like games while building a database of facial image data enriched for a range of emotions exhibited by the child during the game sessions.

The primary contributions of this study are as follows:

1. We present a mobile charades game, Guess What?, to crowdsource emotive video from its players. This framework has utility both as a mechanism for the evaluation of existing emotion classifiers and for the development of novel systems that appropriately generalize to the population of interest.

2. We present a study in which 2602 emotive frames are derived from 21 children with a parent-reported diagnosis of autism using data from the Guess What mobile game collected in a variety of heterogeneous environments.

3. The data were used to evaluate the accuracy and performance of several state-of-the-art classifiers using the workflow shown in Figure 1, to develop an understanding of the feasibility of using these APIs in future mobile therapy approaches. 
Figure 1. A mobile charades game played between caregiver and child is used to crowdsource emotive video, subsampled and categorized by both manual raters and automatic classifiers. Frames from these videos form the basis of our dataset to evaluate several emotion classifiers.

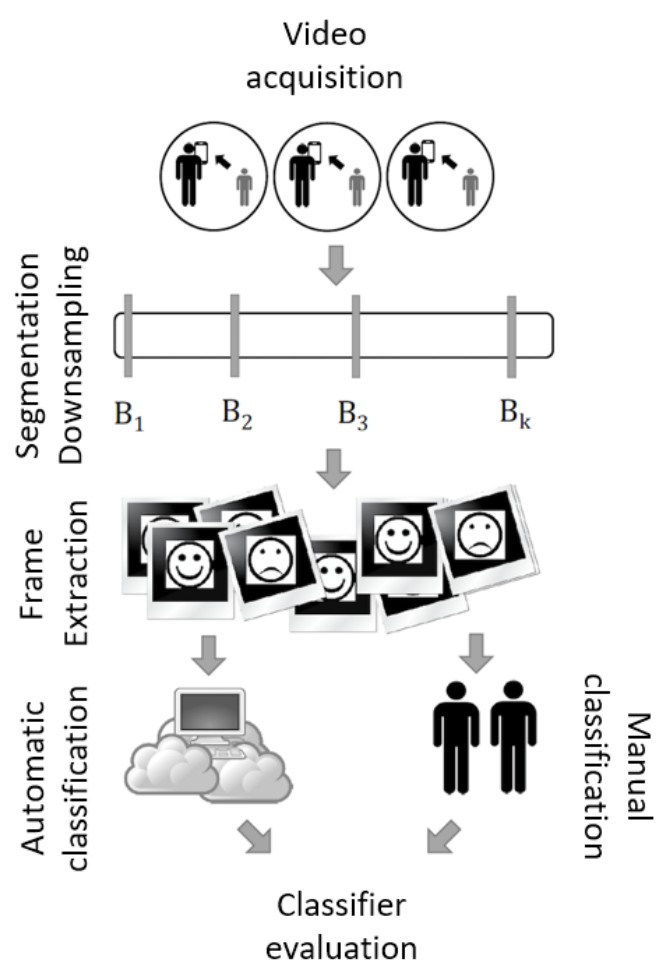

\section{Related Work}

To the best of our knowledge, this is the first work to date that benchmarks public emotion recognition APIs on children with developmental delays. However, a number of interesting apps have been proposed in recent years, which employ vision-based tools or affective computing solutions as an aid for children with autism. The emergence of these approaches motivates a careful investigation of the feasibility of commercial emotion classification algorithms for the pediatric population.

Motivated by the fact that children with autism can experience cognitive or emotional overload, which may compromise their communication skills and learning experience, Picard et al [38] provided an overview of technological advances for sensing autonomic nervous system activation in real-time, including wearable electrodermal activity sensors. A more general overview of the role of affective computing in autism is provided by Kalioby et al [39], with the motivating examples of using technology to help individuals better navigate the socioemotional landscape of their daily lives. Among the enumerated devices include those developed at the Massachusetts Institute of Technology media laboratory, such as expression glasses that discriminate between several emotions, skin conductance-sensing gloves for stress detection, and a pressure-sensitive mouse to infer affective state from how individuals interact with the device. Devices made by industry include the SenseWear Pro2 armband, which includes a variety of wearable sensors that can be repurposed for stress and productivity detection, smart gloves that can detect breathing rate and blood pressure, and wireless heart-rate monitors that can be analyzed in the context of environmental stressors [40].
Prior research conducted by us has demonstrated the efficacy of mobile video phenotyping approaches for children with ASD in general [41-47] and via the use of emotion classifiers integrated with the Google Glass platform to provide real-time behavioral support to children with ASD [17-25]. In addition, other studies have confirmed the usability, acceptance, and overall positive impact on families of Google Glass-based systems that use emotion recognition technology to aid social-emotional communication and interaction for autistic children [48,49]. In addition to these efforts, a variety of other smart-glass devices have been proposed. For example, the SenseGlass [50] is among the earliest works that propose leveraging the Google Glass platform to capture and process real-time affective information using a variety of sensors. The authors proposed apps, including the development of affect-based user interfaces, and empowering wearers toward behavioral change through emotion management interventions.

Glass-based affect recognition that predates the Google Glass platform has also been proposed. Scheirer et al [51] used piezoelectric sensors to detect expressions such as confusion and interest, which were detected with an accuracy of $74 \%$. A more recent work proposes a device called Empathy Glasses [52] in which users can see, hear, and feel from the perspective of another individual. The system consists of wearable hardware to transmit the wearers' gaze and facial expression and a remote interface where visual feedback is provided, and data are viewed.

The research for smart-glass-based interventions is further supported by other technological systems that have been developed and examined within the context of developmental delays, including the use of augmented reality for object discrimination training [53], assistive robotics for therapy [54-56], and mobile assistive technologies for real-time social 
skill learning [57]. Furthermore, the use of computer vision and gamified systems to both detect and teach emotions continues to progress. A computational approach to detect facial expressions optimized for mobile platforms was proposed [58], which demonstrated an accuracy of $95 \%$ from a 6-class set of expressions. Leo et al [59] proposed a computational approach to assess the ability of children with ASD to produce facial expressions using computer vision, validated by three expert raters. Their findings demonstrated the feasibility of a human-in-the-loop computer vision system for analyzing facial data from children with ASD. Similar to this study, which utilizes Guess What?, a charades-style mobile game to collect emotional face data, Park and colleagues proposed six game design methods for the development of game-driven frameworks in teaching emotions to children with ASD, of which include: observation, understanding, mimicking, and generalization, and supports the use of game play to produce data of value to computer vision approaches for children with autism [60].

Although not all of the aforementioned research studies employ emotion recognition models directly, they are indicative of a general transition from traditional health care practices to modern mobile and digital solutions that leverage recent advances in computer vision, augmented reality, robotics, and artificial intelligence [61]. Thus, the trend motivates our investigation of the efficacy of state-of-the-art vision models on populations with developmental delay.

\section{Methods}

\section{Overview}

In this section, we describe the architecture of Guess What? followed by a description of the methods employed to obtain test data and processing the frames therein to evaluate the performance of several major emotion classifiers. Although dozens of APIs are available, we limit our analysis to some of the most popular systems from major providers of cloud services as a fair representation of the state-of-the-art in publicly available emotion recognition APIs. The systems evaluated in this work were Microsoft Azure Emotion API (Azure) [26], Amazon AWS Rekognition (AWS) [27], Google Cloud Vision API (Google) [28], and Sighthound (SH) [29].

\section{System Architecture}

The evaluation of the state-of-the-art in public emotion classification APIs on children with ASD requires a dataset derived from subjects from the relevant population group with a fair amount of consistency in its format and structure. Moreover, as data are limited, it is critical that the video contains a high density of emotive frames to simplify the manual annotation process when establishing a ground truth. Therefore, we have developed and launched an educational mobile game on the Google Play Store [34] and iOS App Store [35], Guess What?, from which we derive emotive video.

In this game, parents hold the phone such that the front camera and screen are facing outward toward the child. When the game session begins, the child is shown a prompt that the caregiver must guess based on the child's gestures and facial expressions. After a correct guess is acknowledged, the parent tilts the phone forward, indicating that a point should be awarded. At this time, another prompt is shown. If the one holding the phone cannot make a guess, he/she will tilt the phone backward to skip the frame and automatically proceed to the next. This process repeats until the 90-second game session has elapsed. Meta information is generated for each game session that indicates the times at which various prompts are shown and when the correct guesses occur.

Although a number of varied prompts are available, the two that are most germane to facial affect recognition and emotion recognition are emojis and faces, as shown in Figures 2 and 3, respectively. After the game session is complete, caregivers can elect to share their files and associated metadata to an institution review board-approved secure Amazon S3 bucket that is fully compliant with the Stanford University's high-risk application security standards. A more detailed discussion of the mechanics and applications of Guess What? is described in [29-32].

Figure 2. Prompts from the emoji category are caricatures, but many are still associated with the classic Ekman universal emotions.

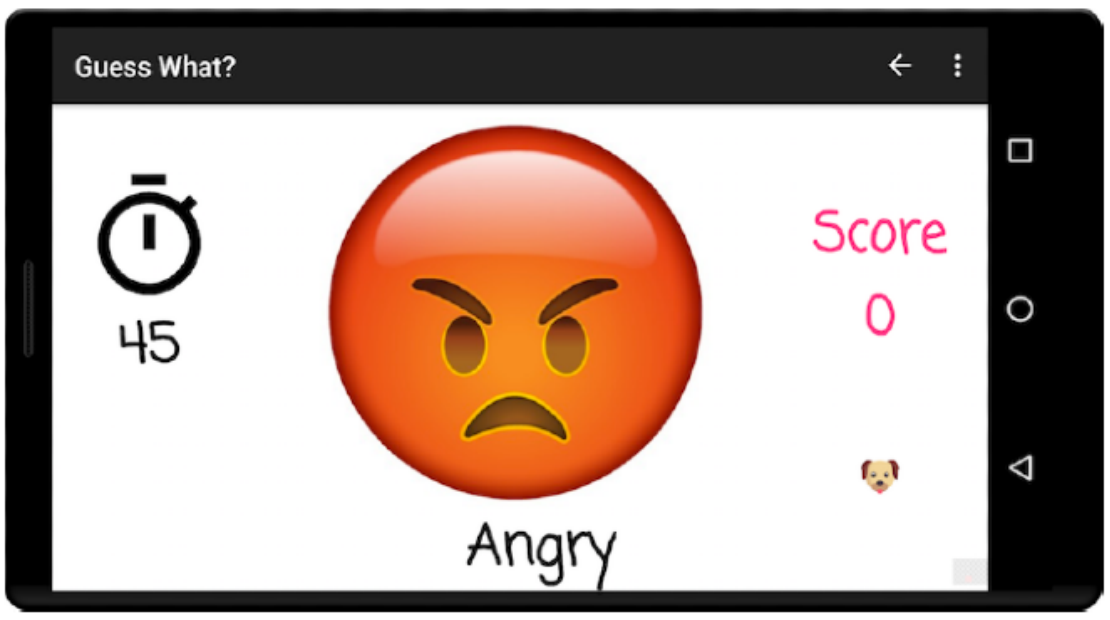


Figure 3. Prompts from the faces category are derived from real photos of children over a solid background.

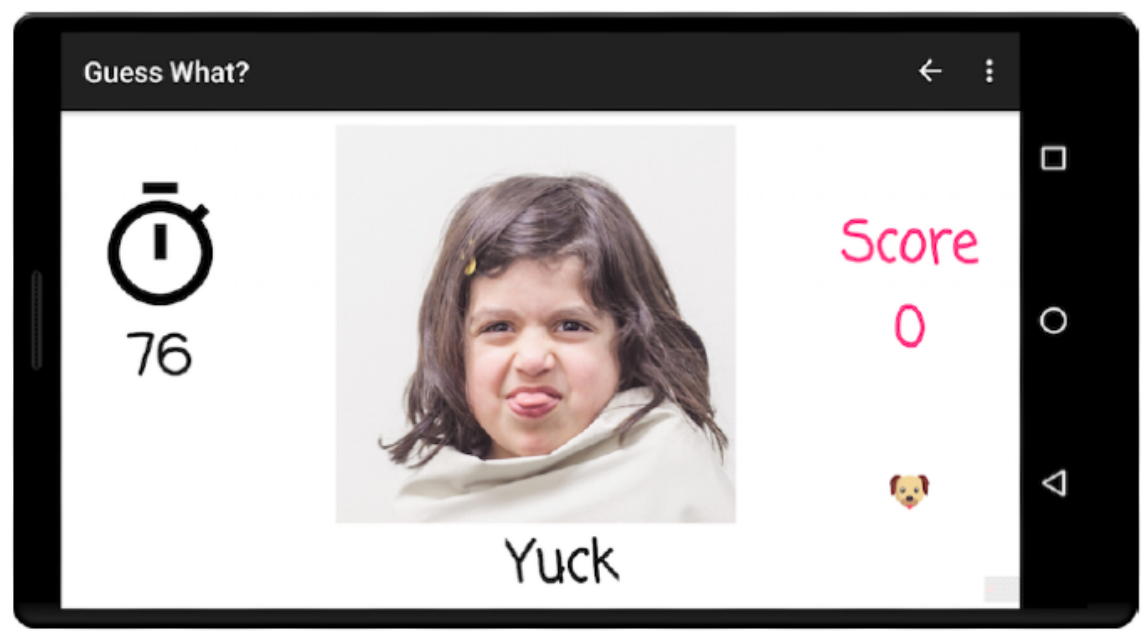

The structure of a video is shown in Figure 4. Each uploaded video yields $n$ video frames, delineated by $k$ boundary points, $B_{1}-B_{k}$, where each boundary point represents the time at which a new prompt is shown to the user. To obtain frames associated with a particular emotion, one should first identify the boundary point associated with that emotion through the game meta information, $i$. Having identified this boundary point, frames between $B_{i}$ and $B_{i}+1$ can be associated with this prompt.
However, two additional factors remain. It typically takes some time, $\alpha$, for the child to react after the prompt is shown. Moreover, there is often a time period, $\beta$, between the child's acknowledgment of the parents' guess and phone tilt by the parent, during which time the child may adopt a neutral facial expression. Therefore, the frames of interest are those that lie between $B_{i}+\alpha$ and $B_{i+1}-\beta$.

Figure 4. The structure of a single video is characterized by its boundary points, which identify the times at which various prompts were shown to the child.

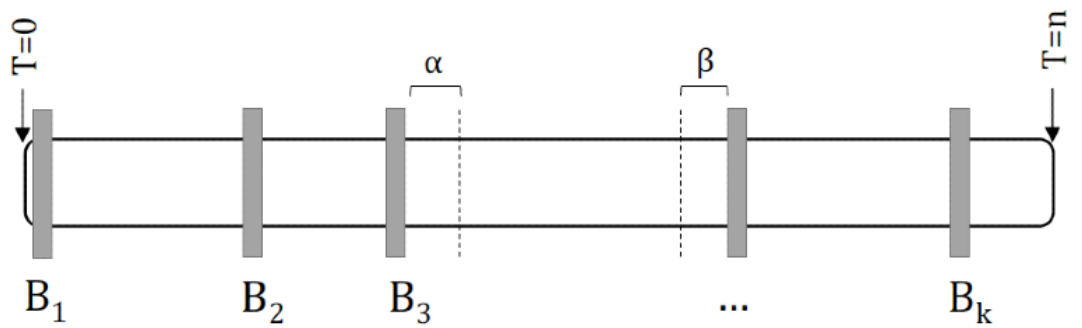

The proposed system is centered on two key aims. First, this mechanism facilitates the acquisition of structured emotive videos from children in a manner that challenges their ability to express facial emotion. Whereas other forms of video capture could be employed, a gamified system encourages repeated use and has the potential to contain a much higher density of emotive frames than a typical home video structured around nongaming activities. As manual annotation is employed as a ground truth for evaluating emotion classification, a high concentration of emotive frames within a short time period is essential to the simplification and reduction of the burden associated with this process. A second aim is to potentially facilitate the aggregation of labeled emotive videos from children using a crowdsourcing mechanism. This can be used to augment existing datasets with labeled images or create new ones for the development of novel deep-learning-based emotion classifiers that can potentially overcome the limitations of existing methods.

\section{Data Acquisition}

A total of 46 videos from 21 subjects were analyzed in this study. These data were collected over 1 year. Ten videos were

collected in a laboratory environment from six subjects with ASD who played several games in a single session administered by a member of the research staff. An additional 36 videos were acquired through crowdsourcing from 15 remote participants. Diagnosis of any form of developmental disorder was provided by the caregiver through self-report during the registration process, along with demographic information (gender, age, ethnicity). The collected information included diagnoses of autistic disorder (autism), ASD, Asperger's syndrome, pervasive developmental disorder (not otherwise specified), childhood disintegrative disorder, no diagnosis, no diagnosis but suspicious, and social communication (pragmatic) disorder. Additionally, a free-text field was available for parents to specify additional conditions. The videos were evaluated by a clinical professional using the Diagnostic and Statistical Manual of Mental Disorders-V criteria before inclusion [1]. Caregivers of all children who participated in the study selected the autism spectrum disorder option.

The format of a Guess What? gameplay session generally enforces a structure on the derived video: the device is held in landscape mode, the child's face is contained within the frame, 
and the distance between the child and camera is typically between 2 and 10 feet. Nevertheless, these videos were carefully screened by members of the research staff to ensure the reliability and quality of the data therein; videos that did not include children, were corrupt, filmed under poor lighting conditions, or did not include plausible demographic information were excluded from the analysis. The average age of the participating children was 7.3 (1.76) years. Due to the small sample size and nonuniform incidence of autism between genders [62], 18 of the 21 participants were male. Although participants explored a variety of game mechanics, all analyzed videos were derived from the two categories most useful for the study of facial affect: faces and emojis. After each game session, the videos were automatically uploaded to an Amazon S3 bucket through an Android background process.

\section{Data Processing}

Most emotion classification APIs charge users per an http request, rendering the processing of every frame in a video prohibitive in terms of both time and cost. To simplify our evaluation, we subsampled each video at a rate of two frames per second. These frames formed the basis of our experiments. To obtain ground truth, two raters manually assigned an emotion label to each frame based on the seven Ekman universal emotions [63], with the addition of a neutral label. Some frames were discarded when there was no face, or the quality was too poor to make an assessment. A classifier's performance on a frame was evaluated only under the conditions that the frame was valid (of sufficient quality), and the two manual raters agreed on the emotion label associated with the frame. Frames were considered of insufficient quality if: (1) the frame was too blurry to discern, (2) the child was not in the frame, (3) the image or video was corrupt, or (4) there were multiple individuals within the frame.

From a total of 5418 reviewed frames, 718 were discarded due to a lack of agreement between the manual raters. An additional 2123 frames were discarded because at least one rater assigned the not applicable (N/A) label, indicating that the frame was of insufficient quality. This was due to a variety of factors but generally caused by motion artifacts or the child leaving the frame due to the phone being tilted in acknowledgment of a correct guess. The total number of analyzed frames was 2602 divided between the categories shown in Table 1.

As shown, most frames were neutral, with a preponderance of happy frames in the nonneutral category. Owing to the limited number of scared and confused frames, this emotion was omitted from our analysis. We also merged the contempt and anger categories due to their similarity of affect and streamline analysis.

Table 1. The distribution of frames per category ( $\mathrm{N}=2602)$.

\begin{tabular}{ll}
\hline Emotion & Frames, $\mathrm{n}$ \\
\hline Neutral & 1393 \\
Emotive & 1209 \\
Happy & 864 \\
Sad & 60 \\
Surprised & 165 \\
Disgusted & 69 \\
Angry & 51 \\
\hline
\end{tabular}

As not all emotion classifiers represented their outputs in a consistent format, some further simplifications were made in our analysis. First, it was necessary to make minor corrections to the format of the outputted data. For example, happy and happiness were considered identical. In the case of AWS, the confused class was ignored, as many other classifiers did not support it. Moreover, calm was renamed neutral. As AWS, Azure, and Sighthound returned probabilities rather than a single label, a frame in which no emotion class was associated with a probability of over $70 \%$ was considered a failure. For Google Vision, classification confidence was associated with a categorical label rather than a percentage. In this case, frames did not receive an emotion classification as likely or very likely were considered failures. It is also worth noting that this platform, unlike all the others, does not contain disgust or neutral classes. The final emotions evaluated in this study were happy, sad, surprise, anger, disgust, and neutral, with the latter two omitted for Google Cloud Vision.
As real-time use is an important aspect of mobile therapies and aids, we evaluated the performance of each classifier by calculating the number of seconds required to process each 90 -second video subsampled to one frame per second. This evaluation was performed on a Wi-Fi network tested with an average download speed of $51 \mathrm{Mbps}$ and an average upload speed of 62.5 Mbps. For each classifier, this experiment was repeated 10 times to obtain the average amount of time required to process the subsampled video.

\section{Results}

\section{Overview}

In this section, we present the results of our evaluation of Guess What? as well as the performance of the evaluated classifiers: Microsoft Azure Emotion API (Azure) [26], AWS [27], Google Cloud Vision API (Google) [28], and SH [29]. Abbreviations for emotions described within this section can be found in Textbox 1. 
Textbox 1. Abbreviations for emotions.

\author{
HP: Happy \\ CF: Confused \\ N/A: Not applicable \\ SC: Scared \\ SP: Surprised \\ DG: Disgusted \\ AG: Angry
}

\section{Classifier Accuracy}

\section{Comparison With Ground Truth (Classifiers)}

Table 2 shows the performance of each classifier calculated by the percentage of correctly identified frames compared with the ground truth for categories neutral, emotive, and all. A neutral frame is one in which the face is recognized, and the neutral label is assigned high confidence. Any other frame within the categories of happy, sad, surprised, disgusted, and angry, are considered emotive frames. A more detailed breakdown of performance by emotion is shown in Table 3. Note that as before, Google's API does not support the neutral and disgust categories.

Table 2. Percentage of frames correctly identified by classifier: Azure (Azure Cognitive Services), AWS (Amazon Web Services), SH (Sighthound), and Google (Google Cloud Vision). These results only include frames in which there was a face, and the two manual raters agreed on the class. Google Vision API does not support the neutral label.

\begin{tabular}{llll}
\hline Classifier & Frame type & & \\
& Emotive $(\mathrm{n}=1209), \mathrm{n}(\%)$ & Neutral $(\mathrm{n}=1393), \mathrm{n}(\%)$ & All $(\mathrm{n}=2602), \mathrm{n}(\%)$ \\
\hline Azure & $798(66.00)$ & $744(53.40)$ & $1542(59.26)$ \\
AWS $^{\mathrm{a}}$ & $829(68.56)$ & $679(48.74)$ & $1508(57.95)$ \\
Google & $785(64.92)$ & N/A & N/A \\
Sighthound & $664(54.92)$ & $902(64.75)$ & $1566(60.18)$ \\
\hline
\end{tabular}

${ }^{\mathrm{a}}$ AWS: Amazon AWS Rekognition.

${ }^{\mathrm{D}} \mathrm{N} / \mathrm{A}$ : not applicable.

Table 3. Percentage of frames correctly identified by emotion type by each classifier: Azure (Azure Cognitive Services), AWS (Amazon Web Services), SH (Sighthound), and Google (Google Cloud Vision). These results only include frames in which there was a face, and the two manual raters agreed on the class. Note: Google Vision API does not support the neutral or disgust labels.

\begin{tabular}{|c|c|c|c|c|c|c|}
\hline \multirow[t]{2}{*}{ Classifier } & \multicolumn{6}{|l|}{ Frame type } \\
\hline & Neutral $(\mathrm{n}=1394), \mathrm{n}(\%)$ & Happy (n=864), n (\%) & $\mathrm{Sad}(\mathrm{n}=60), \mathrm{n}(\%)$ & $\begin{array}{l}\text { Surprised }(\mathrm{n}=165) \text {, } \\
\mathrm{n}(\%)\end{array}$ & $\begin{array}{l}\text { Disgusted }(\mathrm{n}=69), \\
\mathrm{n}(\%)\end{array}$ & Angry $(\mathrm{n}=51), \mathrm{n}(\%)$ \\
\hline AWS & 679 (48.74) & $709(82.0)$ & $19(31)$ & $94(56.9)$ & $4(5)$ & $3(5)$ \\
\hline Sighthound & $902(64.75)$ & $545(63.0)$ & $13(21)$ & $90(54.5)$ & $10(14)$ & $6(11)$ \\
\hline Azure & $744(53.41)$ & $695(80.4)$ & $20(33)$ & $80(48.4)$ & $0(0)$ & $3(5)$ \\
\hline Google & $\mathrm{N} / \mathrm{A}^{\mathrm{a}}$ & $676(78.2)$ & $10(16)$ & $93(56.3)$ & N/A & $6(11)$ \\
\hline
\end{tabular}

${ }^{\mathrm{a} N} / \mathrm{A}$ : not applicable.

\section{Interrater Reliability (Classifiers)}

The Cohen kappa statistic is a measure of interrater reliability that factors in the percentage of agreement due to chance; an important consideration when the possible classes are few in number. Figure 5 shows the agreement between every pair of classifiers based on their Cohen kappa score calculated based on every evaluated frame, in which a score of 1 indicates perfect agreement. The results reflect low agreement between most combinations of classifiers. This is particularly true for the lack of agreement between Google and Sighthound, with a Cohen kappa score of 0.2. This is likely because of differences in how the classifiers are tuned for precision and recall; Sighthound correctly identified more neutral frames than the others, but performance was lower for the most predominant emotive label: happy. 
Figure 5. The Cohen's Kappa Score is a measure of agreement between two raters, and was calculated for all four evaluated classifiers: Azure (Azure Cognitive Services), AWS (Amazon Web Services), SH (Sighthound), and Google (Google Cloud Vision). Results indicate weak agreement between all pairs of classifiers.

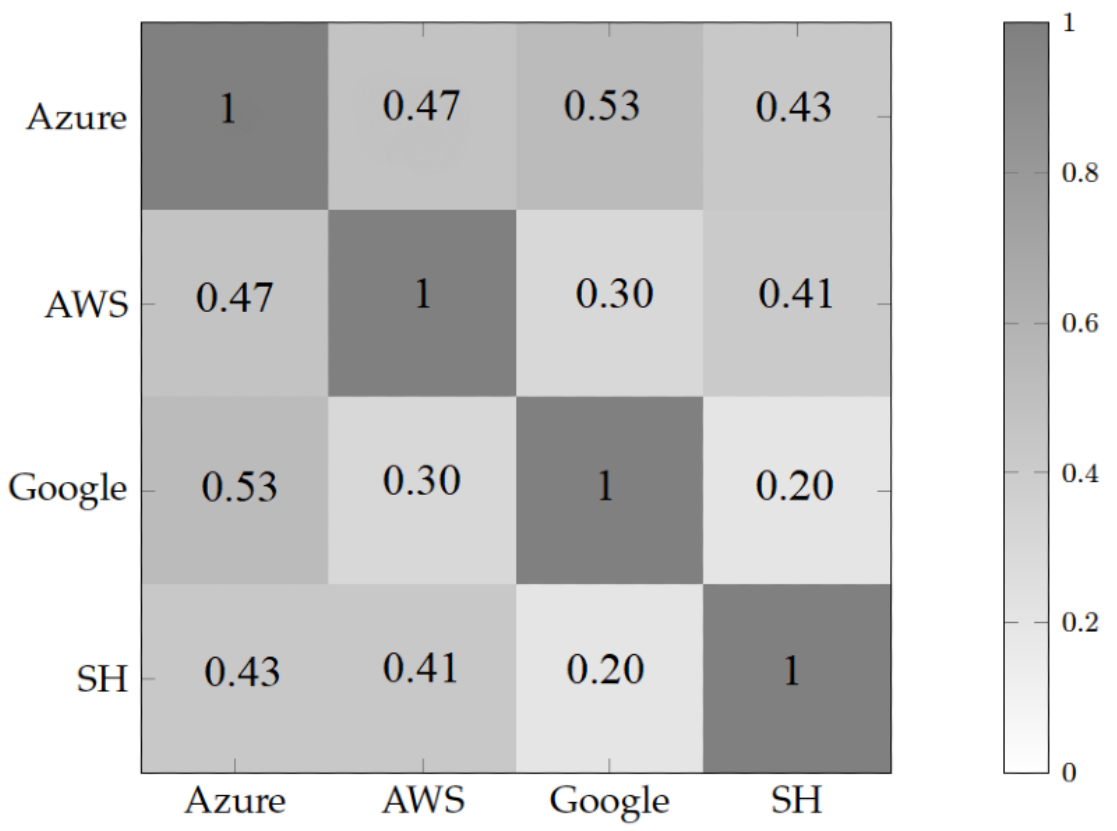

\section{Interrater Reliability (Human Raters)}

The Cohen kappa coefficient for agreement between the two manual raters was 0.74 , which was higher than any combination of automatic classifiers evaluated in this study. Although this indicates substantial agreement, it is worth exploring the characteristics of frames in which there was disagreement between the two raters. The full confusion matrix can be seen in Figure 6, which shows the distribution of all frames evaluated by the raters. The results indicate that most discrepancies were between happy and neutral. These discrepancies were likely subtle differences in how the raters perceived a happy face due to the inherent subjectivity of this process. A lack of agreement can also be seen between the disgust-anger categories.

Figure 6. The distribution of frames between the two human raters for each emotion: HP (Happy), SD (Sad), AG (Angry), DG (Disgust), NT (Neutral), and SC (Scared).

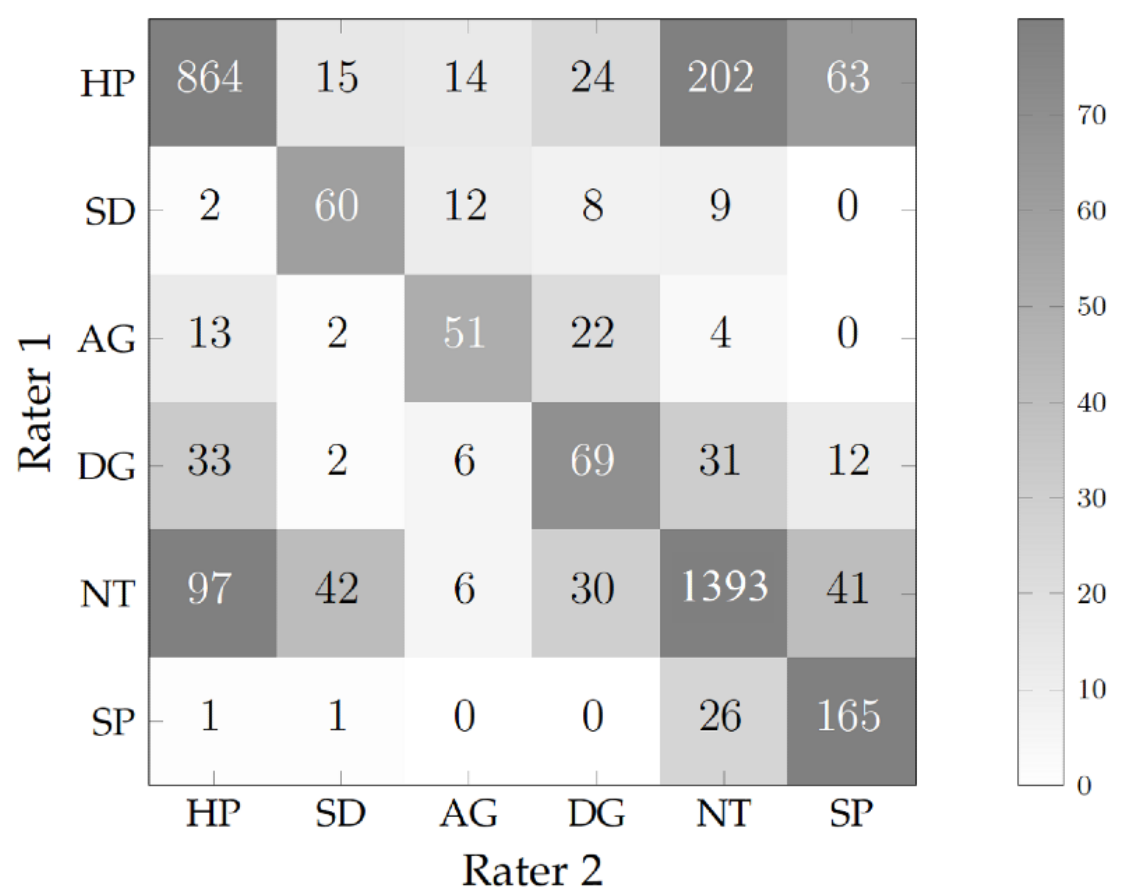

\section{Classifier Speed}

Wearable and mobile solutions for autism generally require efficient classification performance to provide real-time feedback to users. In some cases, this may be environmental feedback, as in the Autism Glass [17-25], which uses the outward-facing camera of Google Glass to read the emotions of those around the child and provide real-time social queues. 
In the case of Guess What? the phone's front camera is used to read the expression of the child, which can be analyzed to determine if the facial expression matches the prompt displayed at that time.

To determine if real-time classification performance is feasible with computation offloaded implementations of emotion classifiers, we measured the amount of time required to process a 90-second video recorded at 30 frames per second and subsampled to one frame per second, yielding a total of 90 frames. For each classifier, this experiment was repeated 10 times to obtain the average number of seconds required to process the subsampled video. Table 4 shows the speed of the API-based classifiers used in this study. The values shown in this table represent the amount of time necessary to send each frame to the Web service via an http post request and receive an http response with the emotion label. These frames were processed sequentially, with no overlap between http requests.

Table 4. Speed of the evaluated classifiers.

\begin{tabular}{ll}
\hline Classifier & Time (seconds) \\
\hline Azure & 28.6 \\
AWS & 90.6 \\
Google & 55.9 \\
Sighthound & 41.1 \\
\hline
\end{tabular}

The findings indicated that the fastest classifier was Azure, processing all 90 frames in a total of 28.6 seconds. Using Azure with a fast internet connection, it may be possible to obtain semi real-time emotion classification performance, a time of 28.6 seconds corresponds to 3.14 frames per second, which is within the bounds of what could be considered real time. The slowest classifier was AWS, which processed these 90 frames in 90.6 seconds. This corresponds to a frame rate of 0.99 frames per second. In summary, real-time or semi-real-time performance is possible with Web-based emotion classifiers on fast Wi-Fi internet connections. For cellular connections or apps that require frame rates beyond three frames per second, these approaches may be insufficient.

\section{Discussion}

\section{Classifier Performance}

Results indicate that Google and AWS produced the highest percentage of correctly classified emotive frames, whereas Sighthound produced the highest percentage of correctly identified neutral frames. Google's API did not provide a neutral label and, therefore, could not be evaluated. The best system in terms of overall classification accuracy was Sighthound by a small margin, with $60.18 \%$ (1566/2602) of the frames correctly identified. Further results indicate that none of the classifiers performed well for any category besides happy, which was the emotion most represented in the dataset, as shown in Table 1. In addition, there appears to be a systematic bias toward high recall and low precision for the happy category: those classifiers that identified most of the happy frames performed worse for those in the neutral category.

In summary, the data suggest that although a frame with a smile will be correctly identified in most cases, the ability of the evaluated classifiers to identify other expressions for children with ASD is dubious and presents an obstacle in the design of emotion-based mobile and wearable outcome measures, screening tools, and therapies.

\section{Analysis of Frames}

Figure 7 shows six frames from one study participant, reproduced with permission from the child's parents. The top of each frame lists the gold-standard annotation in which both raters agreed on a suitable label for the frame. The bottom of each frame enumerates the labels assigned from each classifier in order: Amazon Rekognition, Sighthound, Azure Cognitive Services, and Google Cloud Vision AI. It should be noted that, as before, these labels are normalized for comparison because each classifier outputs data in a particular format. For example, $N / A$ from one classifier could be compared with a blank field in another, whereas some such as Google Cloud explicitly state Not Sure; for our purposes, all three of these scenarios were labeled as N/A during analysis.

Frame A shows a frame that was labeled as neutral by the raters, although each classifier provided a different label: confused, disgusted, happy, and N/A. This is an example of a false-positive, detecting an emotion in a neutral frame. A similar example is shown in frame F; most classifiers failed to identify the neutral label. Such false positives are particularly problematic as the neutral label is the most prevalent, as shown in Table 1.

In contrast, frames $\mathrm{B}$ and $\mathrm{C}$ are examples in which the labels assigned by each classifier matched the labels assigned by the manual raters; all classifiers correctly identified the happy label. As shown in Table 1, happy was the most common nonneutral emotion by a considerable margin, and most classifiers performed quite well in this category; AWS, Azure, and Sighthound all correctly identified between 78.2\% (676/864) and $82.0 \%$ (709/864) of these frames, although at the expense of increased false-positives such as those shown in frames A and F. An example of a happy frame that was identified as such by the human raters but incorrectly by most classifiers is frame D. It is possible that the child's hands covering part of her face contribute to this error, as the frame is otherwise quite similar to frame B. Finally, frame $\mathrm{E}$ is an example of a frame that was processed by the classifiers but not included in our experimental results because the human raters flagged the frame as insufficient 
due to motion artifacts. In this case, all four classifiers correctly determined that the frame could not be processed.

Figure 7. A comparison of the performance of each classifier on a set of frames highlights scenarios that may lead to discrepancies in the classifier outputs for various emotions: HP (Happy), CF (Confused), DG (Disgust), N/A (Not Applicable), AG (Angry), SC (Scared). Ground truth manual labels are shown on top, with labels derived from each classifier on the bottom.

A. Neutral

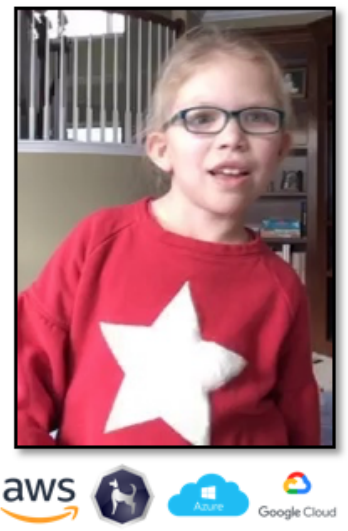

$\mathrm{CF} \cdot \mathrm{DG} \cdot \mathrm{HP} \cdot \mathrm{N} / \mathrm{A}$

D. Happy

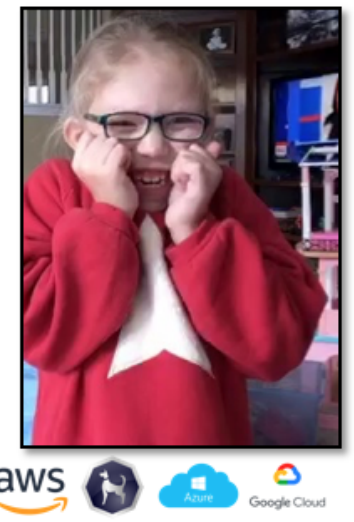

$\mathrm{HP} \cdot \mathrm{AG} \cdot \mathrm{N} / \mathrm{A} \cdot \mathrm{N} / \mathrm{A}$
B. Happy

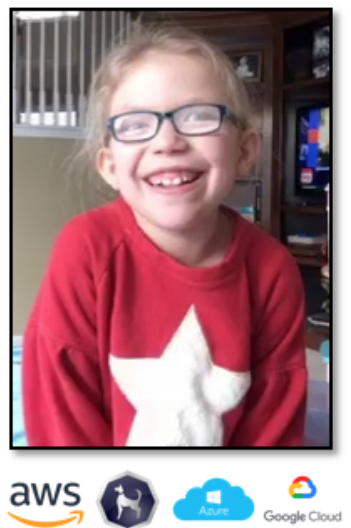

$\mathrm{HP} \cdot \mathrm{HP} \cdot \mathrm{HP} \cdot \mathrm{HP}$

E. N/A

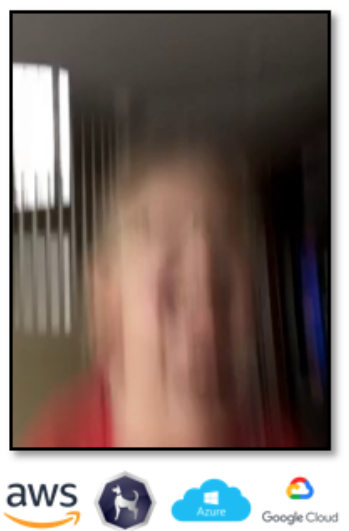

$N / A \cdot N / A \cdot N / A \cdot N / A$
C. Happy

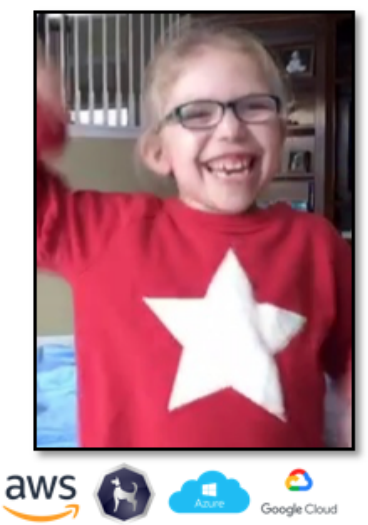

$\mathrm{HP} \cdot \mathrm{HP} \cdot \mathrm{HP} \cdot \mathrm{HP}$

F. Neutral

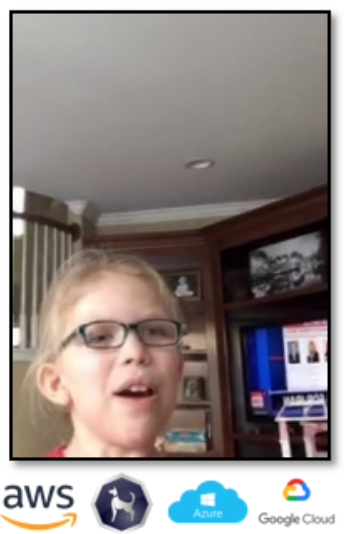

$\mathrm{CF} \cdot \mathrm{SC} \cdot \mathrm{HP} \cdot \mathrm{N} / \mathrm{A}$

\section{Limitations}

There are several limitations associated with this study, which will be addressed in future work. First, we analyzed only a subset of existing emotion classifiers, emphasizing those from providers of major cloud services. Future efforts will extend this evaluation to include those that are less prolific and require paid licenses. A second limitation is the use of parent-reported diagnoses, which may not always be factual. A third limitation is that although we ruled out some comorbid conditions, we did not rule out all comorbid conditions, including Attention-Deficit/Hyperactivity Disorder, which has been shown to impact emotional processing and function in children [64]. A fourth limitation stems from the lack of neurotypical children. Finally, the dataset we used included an unequal distribution of frames across emotion categories. In the future, we will investigate ways to gather equal numbers of frames, and if this distribution may be related to social deficits associated with autism, or increased prevalence of happy and neutral due to the inherent nature of gameplay. Although our results support the conclusion that the commercial emotion classifiers tested here are not yet at a level needed for use with autistic children, it remains unclear how these models will perform with a larger, more diverse, and stratified sample.

\section{Conclusions}

In this feasibility study, we evaluated the performance of four emotion recognition classifiers on children with ASD: Google Cloud Vision, Amazon Rekognition, Microsoft Azure Emotion API, and Sighthound. The average percentage of correctly identified emotive and neutral frames for all classifiers combined was $63.60 \%$ (769/1209) and 55.63\% (775/1393), respectively, varying greatly between classifiers based on how their sensitivity and specificity were tuned. The results also demonstrated that while most classifiers were able to consistently identify happy frames, the performance for sad, disgust, and anger was poor: no classifier identified more than one-third of the frames from either of these categories. We conclude that the performance of the evaluated classifiers is not yet at the level for use in mobile and/or wearable therapy solutions for autistic children, necessitating the development of larger training datasets from these populations to develop more domain-specific models. 


\section{Acknowledgments}

This work was supported in part by funds to DW from the National Institute of Health (1R01EB025025-01 and 1R21HD091500-01), the Hartwell Foundation, Bill and Melinda Gates Foundation, Coulter Foundation, and program grants from Stanford's Human Centered Artificial Intelligence Program, Precision Health and Integrated Diagnostics Center, Beckman Center, Bio-X Center, Predictives and Diagnostics Accelerator Spectrum, the Wu Tsai Neurosciences Institute Neuroscience: Translate Program, Stanford Spark, and the Weston Havens Foundation. We also acknowledge generous support from Bobby Dekesyer and Peter Sullivan. Dr Haik Kalantarian would like to acknowledge the support from the Thrasher Research Fund and Stanford NLM Clinical Data Science program (T-15LM007033-35).

\section{Conflicts of Interest}

DW is the founder of Cognoa. This company is developing digital solutions for pediatric behavioral health, including neurodevelopmental conditions such as autism that are detected and treated using techniques, including emotion classification. AK works as a part-time consultant for Cognoa. All other authors declare no competing interests.

\section{References}

1. American Psychological Association. Diagnostic and Statistical Manual of Mental Disorders. Fifth Edition. Arlington, VA: American Psychiatric Pub; 2013.

2. Lozier LM, Vanmeter JW, Marsh AA. Impairments in facial affect recognition associated with autism spectrum disorders: a meta-analysis. Dev Psychopathol 2014 Nov;26(4 Pt 1):933-945. [doi: 10.1017/S0954579414000479] [Medline: 24915526]

3. Loth E, Garrido L, Ahmad J, Watson E, Duff A, Duchaine B. Facial expression recognition as a candidate marker for autism spectrum disorder: how frequent and severe are deficits? Mol Autism 2018;9:7 [FREE Full text] [doi:

10.1186/s13229-018-0187-7] [Medline: 29423133]

4. Fridenson-Hayo S, Berggren S, Lassalle A, Tal S, Pigat D, Bölte S, et al. Basic and complex emotion recognition in children with autism: cross-cultural findings. Mol Autism 2016;7:52 [FREE Full text] [doi: 10.1186/s13229-016-0113-9] [Medline: 28018573]

5. Kogan MD, Vladutiu CJ, Schieve LA, Ghandour RM, Blumberg SJ, Zablotsky B, et al. The prevalence of parent-reported autism spectrum disorder among US children. Pediatrics 2018 Dec;142(6) [FREE Full text] [doi: 10.1542/peds.2017-4161] [Medline: 30478241$]$

6. Rogers SJ. Brief report: early intervention in autism. J Autism Dev Disord 1996 Apr;26(2):243-246. [doi:

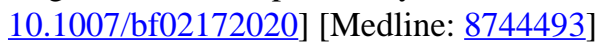

7. Cooper JO, Heron TE, Heward WL. Applied Behavior Analysis. New Jersey: Pearson; 2007.

8. Dawson G, Rogers S, Munson J, Smith M, Winter J, Greenson J, et al. Randomized, controlled trial of an intervention for toddlers with autism: the Early Start Denver Model. Pediatrics 2010 Jan;125(1):e17-e23 [FREE Full text] [doi: 10.1542/peds.2009-0958] [Medline: 19948568 ]

9. Dawson G. Early behavioral intervention, brain plasticity, and the prevention of autism spectrum disorder. Dev Psychopathol 2008;20(3):775-803. [doi: 10.1017/S0954579408000370] [Medline: 18606031]

10. Dawson G, Jones EJ, Merkle K, Venema K, Lowy R, Faja S, et al. Early behavioral intervention is associated with normalized brain activity in young children with autism. J Am Acad Child Adolesc Psychiatry 2012 Nov;51(11):1150-1159 [FREE Full text] [doi: 10.1016/j.jaac.2012.08.018] [Medline: 23101741]

11. Dawson G, Bernier R. A quarter century of progress on the early detection and treatment of autism spectrum disorder. Dev Psychopathol 2013 Nov;25(4 Pt 2):1455-1472. [doi: 10.1017/S0954579413000710] [Medline: 24342850]

12. Scherzer AL, Chhagan M, Kauchali S, Susser E. Global perspective on early diagnosis and intervention for children with developmental delays and disabilities. Dev Med Child Neurol 2012 Dec;54(12):1079-1084 [FREE Full text] [doi: 10.1111/j.1469-8749.2012.04348.x] [Medline: 22803576]

13. van Cong T, Weiss B, Toan KN, Le Thu TT, Trang NT, Hoa NT, et al. Early identification and intervention services for children with autism in Vietnam. Health Psychol Rep 2015;3(3):191-200 [FREE Full text] [doi: 10.5114/hpr.2015.53125] [Medline: 27088123]

14. Samms-Vaughan ME. The status of early identification and early intervention in autism spectrum disorders in lower- and middle-income countries. Int J Speech Lang Pathol 2014 Feb;16(1):30-35. [doi: 10.3109/17549507.2013.866271] [Medline: 24397842]

15. Mandell DS, Novak MM, Zubritsky CD. Factors associated with age of diagnosis among children with autism spectrum disorders. Pediatrics 2005 Dec;116(6):1480-1486 [FREE Full text] [doi: 10.1542/peds.2005-0185] [Medline: 16322174]

16. Ning M, Daniels J, Schwartz J, Dunlap K, Washington P, Kalantarian H, et al. Identification and quantification of gaps in access to autism resources in the United States: an infodemiological study. J Med Internet Res 2019 Jul 10;21(7):e13094 [FREE Full text] [doi: 10.2196/13094] [Medline: $\underline{\text { 31293243] }}$

17. Washington P, Wall D, Voss C, Kline A, Haber N, Daniels J, et al. SuperpowerGlass: A Wearable Aid for the At-Home Therapy of Children with Autism. In: Proceedings of the ACM on Interactive, Mobile, Wearable and Ubiquitous Technologies. 2017 Presented at: ACM'17; June 26-30, 2017; Cambridge, MA. [doi: 10.1145/3130977] 
18. Washington P, Catalin V, Nick H, Serena T, Jena D, Carl F, et al. A Wearable Social Interaction Aid for Children with Autism. In: Proceedings of the 2016 CHI Conference Extended Abstracts on Human Factors in Computing Systems. 2016 Presented at: CHI EA'16; May 7-12, 2016; California, USA p. 2348-2354. [doi: 10.1145/2851581.2892282]

19. Daniels J, Schwartz J, Haber N, Voss C, Kline A, Fazel A, et al. 5.13 Design and efficacy of a wearable device for social affective learning in children with autism. J Am Acad Child Psy 2017 Oct;56(10):S257. [doi: 10.1016/j.jaac.2017.09.296]

20. Daniels J, Schwartz JN, Voss C, Haber N, Fazel A, Kline A, et al. Exploratory study examining the at-home feasibility of a wearable tool for social-affective learning in children with autism. NPJ Digit Med 2018;1:32 [FREE Full text] [doi: 10.1038/s41746-018-0035-3] [Medline: 31304314]

21. Daniels J, Haber N, Voss C, Schwartz J, Tamura S, Fazel A, et al. Feasibility testing of a wearable behavioral aid for social learning in children with autism. Appl Clin Inform 2018 Jan;9(1):129-140 [FREE Full text] [doi: 10.1055/s-0038-1626727] [Medline: 29466819]

22. Voss C, Schwartz J, Daniels J, Kline A, Haber N, Washington P, et al. Effect of wearable digital intervention for improving socialization in children with autism spectrum disorder: a randomized clinical trial. JAMA Pediatr 2019 May 1;173(5):446-454 [FREE Full text] [doi: 10.1001/jamapediatrics.2019.0285] [Medline: 30907929]

23. Voss C, Washington P, Haber N, Kline A, Daniels J, Fazel A, et al. Superpower Glass: Delivering Unobtrusive Real-Time Social Cues in Wearable Systems. In: Proceedings of the 2016 ACM International Joint Conference on Pervasive and Ubiquitous Computing. 2016 Presented at: UbiComp'16; September 12-16, 2016; Heidelberg, Germany p. 1218-1226. [doi: $\underline{10.1145 / 2968219.2968310}]$

24. Voss C, Haber N, Wall DP. The potential for machine learning-based wearables to improve socialization in teenagers and adults with autism spectrum disorder-reply. JAMA Pediatr 2019 Sep 9 [Online ahead of print]. [doi: 10.1001/jamapediatrics.2019.2969] [Medline: 31498377]

25. Kline A, Voss C, Washington P, Haber N, Schwartz H, Tariq Q, et al. Superpower glass. GetMobile 2019 Nov 14;23(2):35-38. [doi: $10.1145 / 3372300.3372308]$

26. Microsoft Azure. Face: An AI Service That Analyzes Faces in Images URL: https://azure.microsoft.com/en-us/services/ cognitive-services/face/ [accessed 2020-03-17]

27. Amazon Web Services (AWS) - Cloud Computing Services. Amazon Rekognition URL: https://aws.amazon.com/rekognition/ [accessed 2018-06-17]

28. Google Cloud: Cloud Computing Services. Vision AI URL: https://cloud.google.com/vision/ [accessed 2018-06-17]

29. Sighthound. 2018 Jun 17. Detection API \& Recognition API URL: https://www.sighthound.com/products/cloud [accessed 2018-06-17]

30. Cohn J. The Robotics Institute Carnegie Mellon University. 1999. Cohn-Kanade AU-Coded Facial Expression Database URL: https://www.ri.cmu.edu/project/cohn-kanade-au-coded-facial-expression-database/ [accessed 2020-03-24]

31. Sneddon I, McRorie M, McKeown G, Hanratty J. The Belfast induced natural emotion database. IEEE Trans Affective Comput 2012 Jan;3(1):32-41. [doi: 10.1109/t-affc.2011.26]

32. Kalantarian H, Washington P, Schwartz J, Daniels J, Haber N, Wall DP. Guess what? J Healthc Inform Res 2018 Oct;3(1):43-66. [doi: 10.1007/s41666-018-0034-9]

33. Kalantarian H, Washington P, Schwartz J, Daniels J, Haber N, Wall D. A Gamified Mobile System for Crowdsourcing Video for Autism Research. In: Proceedings of the 2018 IEEE International Conference on Healthcare Informatics. 2018 Presented at: ICHI'18; June 4-7, 2018; New York, NY, USA p. 350-352. [doi: 10.1109/ichi.2018.00052]

34. Kalantarian H, Jedoui K, Washington P, Tariq Q, Dunlap K, Schwartz J, et al. Labeling images with facial emotion and the potential for pediatric healthcare. Artif Intell Med 2019 Jul;98:77-86 [FREE Full text] [doi: 10.1016/j.artmed.2019.06.004] [Medline: 31521254$]$

35. Kalantarian H, Jedoui K, Washington P, Wall DP. A mobile game for automatic emotion-labeling of images. IEEE Trans Games 2018:1 epub ahead of print. [doi: 10.1109/tg.2018.2877325]

36. Google Play. Guess What? URL: https://play.google.com/store/apps/details?id=walllab.guesswhat [accessed 2018-06-01]

37. Apple Store. Guess What? (Wall Lab) URL: https://apps.apple.com/us/app/guess-what-wall-lab/id1426891832 [accessed 2020-03-18]

38. Picard RW. Future affective technology for autism and emotion communication. Philos Trans R Soc Lond B Biol Sci 2009 Dec 12;364(1535):3575-3584 [FREE Full text] [doi: 10.1098/rstb.2009.0143] [Medline: 19884152]

39. el Kaliouby R, Picard R, Baron-Cohen S. Affective computing and autism. Ann N Y Acad Sci 2006 Dec;1093:228-248. [doi: 10.1196/annals.1382.016] [Medline: 17312261]

40. Andre D, Pelletier R, Farringdon J, Er S, Talbott WA, Phillips PP, et al. Semantic Scholar. 2006. The Development of the SenseWear Armband, A Revolutionary Energy Assessment Device to Assess Physical Activity and Lifestyle URL: https:/ /www.semanticscholar.org/paper/The-Development-of-the-SenseWear-\%C2\%AE-armband-\%2C-a-to-Andre-Pelletier/ e9e115cb6f381a706687982906d45ed28d40bbac [accessed 2020-03-24]

41. Tariq Q, Daniels J, Schwartz JN, Washington P, Kalantarian H, Wall DP. Mobile detection of autism through machine learning on home video: A development and prospective validation study. PLoS Med 2018 Nov;15(11):e1002705 [FREE Full text] [doi: 10.1371/journal.pmed.1002705] [Medline: 30481180] 
42. Tariq Q, Fleming SL, Schwartz JN, Dunlap K, Corbin C, Washington P, et al. Detecting developmental delay and autism through machine learning models using home videos of Bangladeshi children: Development and validation study. J Med Internet Res 2019 Apr 24;21(4):e13822 [FREE Full text] [doi: 10.2196/13822] [Medline: $\underline{31017583}$ ]

43. Washington P, Kalantarian H, Tariq Q, Schwartz J, Dunlap K, Chrisman B, et al. Validity of online screening for autism: crowdsourcing study comparing paid and unpaid diagnostic tasks. J Med Internet Res 2019 May 23;21(5):e13668 [FREE Full text] [doi: 10.2196/13668] [Medline: $\underline{31124463}$ ]

44. Washington P, Paskov KM, Kalantarian H, Stockham N, Voss C, Kline A, et al. Feature selection and dimension reduction of social autism data. Pac Symp Biocomput 2020;25:707-718 [FREE Full text] [Medline: 31797640]

45. Abbas H, Garberson F, Glover E, Wall DP. Machine learning approach for early detection of autism by combining questionnaire and home video screening. J Am Med Inform Assoc 2018 Aug 1;25(8):1000-1007. [doi: 10.1093/jamia/ocy039] [Medline: 29741630]

46. Levy S, Duda M, Haber N, Wall DP. Sparsifying machine learning models identify stable subsets of predictive features for behavioral detection of autism. Mol Autism 2017;8:65 [FREE Full text] [doi: 10.1186/s13229-017-0180-6] [Medline: 29270283]

47. Fusaro VA, Daniels J, Duda M, DeLuca TF, D'Angelo O, Tamburello J, et al. The potential of accelerating early detection of autism through content analysis of YouTube videos. PLoS One 2014;9(4):e93533 [FREE Full text] [doi:

10.1371/journal.pone.0093533] [Medline: 24740236]

48. Sahin NT, Keshav NU, Salisbury JP, Vahabzadeh A. Second version of Google Glass as a wearable socio-affective aid: positive school desirability, high usability, and theoretical framework in a sample of children with autism. JMIR Hum Factors 2018 Jan 4;5(1):e1 [FREE Full text] [doi: 10.2196/humanfactors.8785] [Medline: 29301738]

49. Keshav NU, Salisbury JP, Vahabzadeh A, Sahin NT. Social communication coaching smartglasses: well tolerated in a diverse sample of children and adults with autism. JMIR Mhealth Uhealth 2017 Sep 21;5(9):e140 [FREE Full text] [doi: 10.2196/mhealth.8534] [Medline: 28935618]

50. Hernandez J, Picard W. SenseGlass: Using Google Glass to Sense Daily Emotions. In: Proceedings of the adjunct publication of the 27th annual ACM symposium on User interface software and technology. 2014 Presented at: UIST'14; October 5 8, 2014; Hawaii USA p. 77-78. [doi: 10.1145/2658779.2658784]

51. Scheirer J, Fernandez R, Picard R. Expression Glasses: A Wearable Device for Facial Expression Recognition. In: Proceedings of the Extended Abstracts on Human Factors in Computing Systems. 1999 Presented at: CHI'99; May 15-20, 1999; Pittsburgh, Pennsylvania p. 262-263. [doi: 10.1145/632716.632878]

52. Katsutoshi M, Kunze K, Billinghurst M. Empathy Glasses. In: Proceedings of the 2016 CHI Conference Extended Abstracts on Human Factors in Computing Systems. 2016 Presented at: CHI EA'16; May 7-12, 2016; San Jose, California p. $1257-1263$. [doi: $\underline{10.1145 / 2851581.2892370]}$

53. Escobedo L, Tentori M, Quintana E, Favela J, Garcia-Rosas D. Using augmented reality to help children with autism stay focused. IEEE Pervasive Comput 2014 Jan;13(1):38-46. [doi: 10.1109/mprv.2014.19]

54. Scassellati B, Admoni H, Matarić M. Robots for use in autism research. Annu Rev Biomed Eng 2012;14:275-294. [doi: 10.1146/annurev-bioeng-071811-150036] [Medline: $\underline{22577778}$ ]

55. Feil-Seifer D, Matarić MJ. Toward socially assistive robotics for augmenting interventions for children with autism spectrum disorders. In: Khatib O, Kumar V, Pappas GJ, editors. Experimental Robotics: The Eleventh International Symposium. Berlin, Heidelberg: Springer; 2009:201-210.

56. Robins B, Dautenhahn K, Boekhorst RT, Billard A. Robotic assistants in therapy and education of children with autism: can a small humanoid robot help encourage social interaction skills? Univ Access Inf Soc 2005 Jul 8;4(2):105-120. [doi: 10.1007/s10209-005-0116-3]

57. Escobedo L, Nguyen DH, Boyd LA, Hirano S, Rangel A, Garcia-Rosas D, et al. MOSOCO: A Mobile Assistive Tool to Support Children With Autism Practicing Social Skills in Real-Life Situations. In: Proceedings of the SIGCHI Conference on Human Factors in Computing Systems. 2012 Presented at: CHI'12; May 5-10, 2012; Austin, USA p. 2589-2598. [doi: 10.1145/2207676.2208649]

58. Palestra G, Pettiniccho A, Del CM, Carcagni P. Improved Performance in Facial Expression Recognition Using 32 Geometric Features. In: Proceedings of the International Conference on Image Analysis and Processing. 2015 Presented at: ICIAP'15; September 7-11, 2015; Genoa, Italy p. 518-528. [doi: 10.1007/978-3-319-23234-8_48]

59. Leo M, Carcagnì P, Distante C, Spagnolo P, Mazzeo P, Rosato A, et al. Computational assessment of facial expression production in ASD children. Sensors (Basel) 2018 Nov 16;18(11) [FREE Full text] [doi: 10.3390/s18113993] [Medline: 30453518]

60. Park JH, Abirached B, Zhang Y. A Framework for Designing Assistive Technologies for Teaching Children With ASDs Emotions. In: Proceedings of the Extended Abstracts on Human Factors in Computing Systems. 2012 Presented at: CHI EA'12; May 5 - 10, 2012; Texas, Austin, USA p. 2423-2428. [doi: 10.1145/2212776.2223813]

61. Washington P, Park N, Srivastava P, Voss C, Kline A, Varma M, et al. Data-driven diagnostics and the potential of mobile artificial intelligence for digital therapeutic phenotyping in computational psychiatry. Biol Psychiatry Cogn Neurosci Neuroimaging 2019 Dec 13 [Online ahead of print]. [doi: 10.1016/j.bpsc.2019.11.015] [Medline: 32085921] 
62. Christensen DL, Baio J, Braun KN, Bilder D, Charles J, Constantino JN, Centers for Disease Control and Prevention (CDC). Prevalence and characteristics of autism spectrum disorder among children aged 8 years--autism and developmental disabilities monitoring network, 11 sites, United States, 2012. MMWR Surveill Summ 2016 Apr 1;65(3):1-23. [doi: 10.15585/mmwr.ss6503a1] [Medline: 27031587]

63. Ekman P, Friesen WV, O'Sullivan M, Chan A, Diacoyanni-Tarlatzis I, Heider K, et al. Universals and cultural differences in the judgments of facial expressions of emotion. J Pers Soc Psychol 1987 Oct;53(4):712-717. [doi:

10.1037//0022-3514.53.4.712] [Medline: 3681648]

64. Sinzig J, Morsch D, Lehmkuhl G. Do hyperactivity, impulsivity and inattention have an impact on the ability of facial affect recognition in children with autism and ADHD? Eur Child Adolesc Psychiatry 2008 Mar;17(2):63-72. [doi: 10.1007/s00787-007-0637-9] [Medline: 17896119]

\author{
Abbreviations \\ ABA: applied behavior analysis \\ AI: artificial intelligence \\ API: application programming interface \\ ASD: autism spectrum disorder \\ ESDM: early start Denver model \\ SH: Sighthound
}

Edited by G Eysenbach; submitted 18.12.18; peer-reviewed by M Leo, N Sahin; comments to author 27.04.19; revised version received
03.07.19; accepted 23.02.20; published 01.04.20
Please cite as:
Kalantarian H, Jedoui K, Dunlap K, Schwartz J, Washington P, Husic A, Tariq Q, Ning M, Kline A, Wall DP
The Performance of Emotion Classifiers for Children With Parent-Reported Autism: Quantitative Feasibility Study
JMIR Ment Health 2020;7(4):e13174
URL: $\underline{\text { https://mental.jmir.org/2020/4/e13174 }}$
doi: $10.2196 / 13174$
PMID: $\underline{32234701}$

CHaik Kalantarian, Khaled Jedoui, Kaitlyn Dunlap, Jessey Schwartz, Peter Washington, Arman Husic, Qandeel Tariq, Michael Ning, Aaron Kline, Dennis Paul Wall. Originally published in JMIR Mental Health (http://mental.jmir.org), 01.04.2020. This is an open-access article distributed under the terms of the Creative Commons Attribution License (https://creativecommons.org/licenses/by/4.0/), which permits unrestricted use, distribution, and reproduction in any medium, provided the original work, first published in JMIR Mental Health, is properly cited. The complete bibliographic information, a link to the original publication on http://mental.jmir.org/, as well as this copyright and license information must be included. 